0148 USING THE UK BIOBANK STUDY TO ESTIMATE OCCUPATIONAL CAUSES OF CHRONIC DISEASE: COMPARABILITY WITH THE UK NATIONAL POPULATION AND ADJUSTMENT FOR BIAS

${ }^{1}$ Sally Hutchings, ${ }^{2}$ Jon Ayres, ${ }^{1}$ Paul Cullinan, ${ }^{3}$ David Fishwick, ${ }^{1}$ Debbie Jarvis, ${ }^{1}$ Sara De Matteis, ${ }^{2}$ Steve Sadhra, ${ }^{1}$ Magda Wheatley, 'Lesley Rushton. ${ }^{1}$ Imperial College London, London, UK; ${ }^{2}$ University of Birmingham, Birmingham, UK; ${ }^{3}$ Health and Safety Laboratory, Buxton, UK

\subsection{6/oemed-2014-102362.245}

Objectives The UK Biobank study is a sample of 502,682 people aged 40-70, clustered around 22 assessment centres. As part of a project to investigate the causes of COPD and estimate the UK occupational burden, we have assessed the sample's representativeness with respect to the UK national population, with a view to accounting for potential biases.

Method We have compared characteristics of the Biobank population (age, education, employment, smoking etc) to that of the UK population as estimated from national data sources.

Results Deprivation index scores indicate that Biobank respondents in more affluent wards are over-represented $(52 \%$ of Biobank respondents versus $28 \%$ nationally have scores of less than -2). The Biobank respondents are also better-educated (33\% to degree level, 17\% nationally), with similar qualification levels in men and women, whereas more men than women nationally in this age range had higher level qualifications. Fewer were currently employed than nationally $(58 \%$ vs. $65 \%)$, particularly men over 60 , with more retired ( $45 \%$ vs. $33 \%$ ), and fewer disabled or unemployed. There are more in managerial and professional (54\% vs. 46\%), and fewer in routine and manual occupations (22\%, 33\% nationally), and fewer smokers (33\% vs. 49\%). Fewer in the already under-represented unskilled occupations ( $47 \%$ vs. $70 \%$ for other occupations), or with reported respiratory ill-health (50\% vs. $59 \%)$ have a usable email address.

Conclusions As Biobank respondents are on average less deprived, better educated and under-represented in unskilled occupations than the national population, estimating national occupational COPD burden, and collecting further data without bias will require data adjustments.

\section{BRAZILIAN CONSTRUCTION INDUSTRY: A CONTRIBUTION TO IMPROVE INFORMATION ON FATAL WORK ACCIDENTS}

Celso Salim. Fundacentro, Belo Horizonte, Minas Gerais State, Brazil

\subsection{6/oemed-2014-102362.246}

Objectives Considering data sources and statistical trends on recent fatal cases resulting from workplace accidents in the Brazilian construction industry, herein two aspects are emphasised: a critical appraisal of the official registers on deaths resulting from work-related accidents, and a discussion on alternatives to achieve better information to support interventions in this sector.

Method Statistics and the variability in the profiles of death at work from 2000 to 2011 were systematically analysed for construction industry according to the federal data sources on dimensions related to occupational health, working conditions and social security.

Results In addition to presentation of specific rates and a set of indicators, a technical proposal is suggested for a more effective and realistic characterisation of the profile of mortality in workplaces by considering age, gender, education, occupation, as well the more frequent causes related to each type of accident. All of them as a condition to understand the main risks and to help teams of OSH facing the worst violence against workers.

Conclusions By reason of the high social and economic costs involving work accidents in Brazil, this study searches to provide a specific comprehension on fatal cases as well as some subsidies for the government to draw policies oriented to minimise risks in the workplaces of construction industry. However, mortality resulting from accidents in the informal labour market should be considered to overcome gap on information. After all, if the number of unemployed workers is underestimated, the number of underemployed has not been taken into account.

\section{ANALYSIS OF WORK RELATED ACCIDENTS IN THE MINING SECTOR 2000-10: THE CASE OF MINAS GERAIS STATE, BRAZIL}

Celso Salim. Fundacentro, Belo Horizonte, Minas Gerais State, Brazil

\subsection{6/oemed-2014-102362.247}

Objectives The purpose of this study is to investigate the trends and differentials of workplace accidents registered in the mining sector in the State of Minas Gerais, located in Southeastern Brazil and responsible for more than $40 \%$ of mineral production in the country. In addition, it intends to identify some restraints to more realistic quantifications and characterizations of workplace accidents as well as to discuss possible alternatives to overcome them.

Method Through a critical view of statistics about fatal and nonfatal accidents at workplace originating from Annual Relation of Social Information and Yearly Workplace Accident Records, it is intended to measure differentials about the accident results i.e. temporary incapacity, death and disability, conceive benefits, lost work time, accident description, ages, sex, instruction degree and occupation.

Results From 2000 to 2010, workplace accidents in the mining sector kept a relatively high level. In fact, they are rising. Except for 2001 and mainly for 2003, when it fell, this trend remained practically unchanged in the period. Yet, this fall may not reflect larger outsourcing and/or an increase in informal work relations. Moreover, accident and death rates or lethality rates place the sector in an uncomfortable rank.

Conclusions The data found in these records can be intrinsically improved and their cover range can be broadened. Even keeping the particularities of mining sector on a regional scale, this work shows a concrete analysis followed by propositions of intervention to achieve a better workplace accidents statistics.

\section{SOCIAL SECURITY IN BRAZIL: THE IMPACT OF EPIDEMIOLOGICAL NEXUS ON THE BENEFITS RELATED TO OCCUPATIONAL DISEASES}

Celso Salim. Fundacentro, Belo Horizonte, Minas Gerais State, Brazil

\subsection{6/oemed-2014-102362.248}

Objectives The Ministry of Social Security, in order to face the under-reporting of occupational accidents and diseases, has introduced new methods of identifying them. In addition to the Employment Accident Notification (CAT), the Technical Epidemiological Social Welfare Nexus (NTEP) was established in 2007. This work intends to analyse the role of the NTEP in 
improving information regarding to the work-related diseases and, more specifically, on the trend and variation of benefits paid for them.

Method Through the NTEP it is possible to establish a nexus for each area of the economic activity, focusing on data of incapacitating diseases recognised by social welfare and involving more than 15 days' absence from work, using the ICD-10.

Results Benefits for work-related diseases increased 128.2\% during 2005-2008. However, the greatest changes occurred after 2007. From May 2006 to March 2007, when only the employer's CAT was used, 125246 Accident and Disease Assistance authorizations were issued. But, with the addition of the NTEP to the CAT this number rose to 293912 , an increase of $134.7 \%$ over the period from April 2007 to February 2008. The detachable figures are for "musculoskeletal system and connective tissue" (107 764 cases), "mental and behavioural disorders" (8930 cases), "and "diseases of the nervous system" (8396 cases).

Conclusions The accident benefits for work-related diseases are growing more than other welfare benefits. This reality requires more studies and technical insights as well as priorities in terms of specific strategies for OSH policy.

\section{ASSOCIATIONS BETWEEN JOB STRESS, SOCIAL SUPPORT AND INSOMNIA AMONG NURSES}

${ }^{1}$ Caroline Kröning Luna, ${ }^{1}$ Lúcia Rotenberg, ${ }^{1}$ Aline Silva-costa, ${ }^{2}$ Susanna Toivanen, ${ }^{3}$ Tânia Araújo, 'Luciana Fernandes Portela, ${ }^{1}$ Rosane Härter Griep. ${ }^{1}$ Education Laboratory for Environment and Health (LEAS), Instituto Oswaldo Cruz - Fiocruz, Rio de Janeiro, Brazil; ${ }^{2}$ Centre for Health Equity Studies, StockholmUniversity/Karolinska Institute, Stockholm, Sweden; ${ }^{3}$ Health Department, State University of Feira de Santana, Feira de Santana, Brazil

\subsection{6/oemed-2014-102362.249}

Objectives To investigate the association between job strain, social support at work and insomnia among registered nurses.

Method A cross-sectional study was conducted among 3.229 nurses $(87 \%$ women) in 18 major public hospitals in Rio de Janeiro/Brazil. Data collection was based on a comprehensive self-filled questionnaire that included questions on insomnia and the Job Content Questionnaire (JCQ). Job strain (high psychological demands and low control) and social support were evaluated by the Portuguese version of the 2.0-JCQ, defined by the quadrant approach. In addition, emotional demands were also assessed, so that high strain was evaluated considering separately the psychological and the emotional demands. Insomnia was defined as having at least one of the insomnia symptoms: difficulty initiating sleep, maintaining sleep or early morning awakening. Multivariate logistic regression and adjusted odds ratios and 95\% confidence intervals were calculated.

Results The overall prevalence of insomnia was 34.3\%. Individuals with high job strain and low social support experienced insomnia more frequently $(\mathrm{p}<0.05)$. After adjusting for sociodemographic, work and health-related variables, high strain doubled the chances of presenting insomnia considering both the psychological $(\mathrm{OR}=2.20, \mathrm{CI} 1.74-2.78)$ and the emotional demands $(\mathrm{OR}=1.99$, CI 1.57-2.53). High strain in combination with low support at work increased the chances of insomnia even more.

Conclusions High strain is suggested as a possible risk factor for insomnia considering both psychological and emotional demands. The lack of social support from co-workers and supervisors seem to potentiate the odds for insomnia.

\section{THE RELATIONSHIP BETWEEN LOW LEVEL BENZENE EXPOSURE AND BLOOD CELL COUNTS IN KOREAN WORKERS}

${ }^{1}$ Dong-Hee Koh, ${ }^{2}$ Hee-Kyung Cheon, ${ }^{2}$ Hyang-Woo Ryu, ${ }^{2}$ Sang-Gil Lee. ${ }^{1}$ Carcinogenic Hazard Brach, National Cancer Control Institute, National Cancer Center, Goyang-Si, Gyeonggi-Do, Republic of Korea; ${ }^{2}$ Occupational Safety and Health Research Institute, Korea Occupational Safety and Health Agency, Incheon, Republic of Korea

\subsection{6/oemed-2014-102362.250}

Objectives Benzene is a well-known haematological toxin causing aplastic anaemia and leukaemia. Recent studies showed that low level benzene less than 1ppm disturbs the hematopoietic system. However, other studies did not show consistent results. The aim of the present study was to examine the relationship between low level benzene exposure and blood cell counts in Korean workers.

Method Blood cell counts of benzene exposed workers were retrieved from a nationwide Worker's Health Examination Database from 2003 to 2008. If a worker did not take a blood test for benzene during 2003-2004, the worker was regarded as a first exposed. Personal air benzene monitoring records were retrieved from nationwide Work Environment Monitoring Database from 2004-2008. Mean benzene levels were calculated and assigned for the various combinations of factory/industry/process codes. Mixed-effects models were employed to examine associations between benzene level, and the numbers of WBC, RBC, platelet, segmented neutrophil, lymphocyte and monocyte.

Results 11281 workers took blood tests during 2005-2008 for the first time. 8679 personal benzene measurements during 2004-2008 across industries were collected. RBC counts showed a significant negative association with low level benzene exposure with a dose-response relationship. WBCs also showed negative association, but did not show a dose-response relationship. Among WBCs, lymphocyte showed a stronger association with low level benzene than other cell types.

Conclusions Our findings support the hematotoxicity of low level benzene exposure. Further study with direct benzene measuring for first exposed workers is needed to confirm the low level benzene toxicity in Korean workers.

\section{DEVELOPMENT OF AN INSTRUMENT ASSESSING SYMPTOM EXAGGERATION IN PATIENTS RECEIVING DISABILITY BENEFITS SECONDARY TO MENTAL HEALTH DISORDERS}

1,2 2 hanil Ebrahim, ${ }^{3}$ Sheena Bance, ${ }^{4}$ Luis Montoya, ${ }^{1}$ Sohail Mulla, ${ }^{1}$ Mostafa Kamal el Din, ${ }^{3}$ Cindy Malachowski, 'Gordon Guyatt, ${ }^{1}$ Busse. ${ }^{1}$ McMaster University, Hamilton, Canada; ${ }^{2}$ Stanford University, Stanford, USA; ${ }^{3}$ University of Toronto, Toronto, Canada; ${ }^{4}$ University Health Network, Toronto, Canada

\subsection{6/oemed-2014-102362.251}

Objectives Symptom exaggeration is a significant issue in patients receiving disability benefits secondary to mental health disorders. Measures designed to detect exaggeration of symptoms are valuable for informing more accurate diagnoses, which can impact claim decision-making, both for disability claim approval and patient management. Our objectives were: 1) to complete a systematic review to identify measures that assess symptom exaggeration in mental health disorders, and 2) using the results from the review, develop an instrument assessing symptom exaggeration in individuals receiving disability benefits secondary to mental health disorders. 